\title{
NeuroImage
}

\section{Brain activity relating to the contingent negative variation: an fMRI investigation}

\author{
Y. Nagai, ${ }^{\mathrm{a}, *}$ H.D. Critchley, ${ }^{\mathrm{b}}$ E. Featherstone, ${ }^{\mathrm{b}}$ P.B.C. Fenwick, ${ }^{\mathrm{c}}$ M.R. Trimble, ${ }^{\mathrm{a}}$ and R.J. Dolan ${ }^{\mathrm{b}}$ \\ a Institute of Neurology, Department of Clinical and Experimental Epilepsy, London WC1N 3BG, UK \\ ${ }^{\mathrm{b}}$ Wellcome Department of Imaging Neuroscience, Institute of Neurology, UCL, London WC1N 3BG, UK \\ ${ }^{\mathrm{c}}$ Institute of Psychiatry, KCL, London SE5 $8 A F$ GB, UK
}

Received 6 May 2003; revised 30 October 2003; accepted 31 October 2003

\begin{abstract}
The contingent negative variation $(\mathrm{CNV})$ is a long-latency electroencephalography (EEG) surface negative potential with cognitive and motor components, observed during response anticipation. $\mathrm{CNV}$ is an index of cortical arousal during orienting and attention, yet its functional neuroanatomical basis is poorly understood. We used functional magnetic resonance imaging (fMRI) with simultaneous EEG and recording of galvanic skin response (GSR) to investigate CNV-related central neural activity and its relationship to peripheral autonomic arousal. In a group analysis, blood oxygenation level dependent (BOLD) activity during the period of CNV generation was enhanced in thalamus, somatomotor cortex, bilateral midcingulate, supplementary motor, and insular cortices. Enhancement of CNVrelated activity in anterior and midcingulate, SMA, and insular cortices was associated with decreases in peripheral sympathetic arousal. In a subset of subjects in whom we acquired simultaneous EEG and fMRI data, we observed activity in bilateral thalamus, anterior cingulate, and supplementary motor cortex that was modulated by trial-by-trial amplitude of CNV. These findings provide a likely functional neuroanatomical substrate for the $\mathrm{CNV}$ and demonstrate modulation of components of this neural circuitry by peripheral autonomic arousal. Moreover, these data suggest a mechanistic model whereby thalamocortical interactions regulate $\mathrm{CNV}$ amplitude.

(C) 2004 Elsevier Inc. All rights reserved.
\end{abstract}

Keywords: Contingent negative variation; Electroencephalography; Blood oxygenation level dependent

\section{Introduction}

The contingent negative variation $(\mathrm{CNV})$ is a slow event-related potential (ERP), generated over several seconds following an eliciting event, observed using electroencephalography (EEG). The CNV is classically induced by forewarned reaction time tasks, where a warning stimulus (S1) is followed by a predictable interval before a second (imperative) stimulus (S2) that cues a reaction time response. The CNV, which develops in the anticipatory interval (between S1

* Corresponding author. Institute of Neurology, Department of Clinical and Experimental Epilepsy, Queen Square, London WC1N 3BG, UK. Fax: +44-20-7278-8772.

E-mail address: y.nagai@ion.ucl.ac.uk (Y. Nagai).

Available online on ScienceDirect (www.sciencedirect.com.) and S2) at the vertex, has been termed the "expectancy wave" (Walter et al., 1964). A more general model of the CNV encapsulates a concept of cortical arousal related to anticipatory attention, preparation, motivation, and information processing (Tecce, 1972).

Neurophysiological studies indicate that cortical surface-negative potentials, such as the $\mathrm{CNV}$, result from depolarization of apical dendrites of cortical pyramidal cells by thalamic afferents and reflect excitation over an extended cortical area (Birbaumer et al., 1990). The CNV itself may reflect integration of dissociable neural processes. The early phase of the $\mathrm{CNV}$, maximal at midline frontal electrodes, encompasses an orienting response to S1. The amplitude of this early component may be modulated by physiological arousal and stimulus properties of S1 (Rohrbaugh et al., 1976). The later component of the CNV, maximal at the vertex, reflects a greater component of motor preparation (Birbaumer et al., 1990; Rockstroh et al., 1991; Rohrbaugh et al., 1976).

It has been proposed that central mechanisms regulating CNV are closely associated with the operation of thalamocortical mechanisms that filter sensory information (Birbaumer et al., 1990; Brunia and Van Boxtel, 2001; Elbert and Rockstroh, 1987; Skinner and Yingling, 1976). Focused attention, necessary for CNV generation, requires the selective filtering of salient sensory information from other distracting sensory inputs to enable specific stimulus-response (S-R) pairings (Yingling and Skinner, 1976). The negative shift in cortical potential underlying the CNV may thus reflect increased thalamocortical sensory information flow (Birbaumer et al., 1990; Brunia and Van Boxtel, 2001; Elbert and Rockstroh, 1987). Consistent with this notion, GABAergic thalamic neuronal activity has been shown to have a modulatory influence on arousal and attention, including activated states of alertness (Le Masson et al., 2002; Steriade, 2001, 2003). However, a relationship between thalamo-cortical interactions and $\mathrm{CNV}$ generation has not been directly demonstrated in humans.

Brain regions including primary motor cortex, anterior cingulate, and supplementary motor area (SMA) are implicated in magnetic field encephalography (MEG) studies as generating the CNV (Ioannides et al., 1994; Liu et al., 1996). However, the contribution of subcortical centers, such as thalamus, to CNVrelated activity has remained largely theoretical. Among cortical regions, the SMA has been implicated in general motor preparation and execution of movements (Lee et al., 1999). However, the 
association of anterior cingulate cortex with $\mathrm{CNV}$ generation is noteworthy since this region is also implicated in autonomic control and integration of peripheral arousal with cognition, for example, during delay in outcome anticipation (Critchley et al., 2000, 2001, 2003). Some studies have demonstrated that CNV amplitude (particularly the early component) may be modulated by peripheral arousal state, for example, increased heart rate (Tecce, 1972), yet the neuroanatomical basis of interactions between CNV and autonomic arousal has not been established.

The present study examines the functional neuroanatomy underlying the $\mathrm{CNV}$ and its interaction with peripheral autonomic arousal. We used functional magnetic resonance imaging (fMRI) to map in detail cortical and subcortical regional activity during $\mathrm{CNV}$ generation. We simultaneously recorded galvanic skin conductance response (GSR) to establish where CNV-related activity was modulated by peripheral sympathetic arousal. To directly establish the relationship between regional brain activity and the CNV, we used simultaneous AMRI and EEG to identify activity correlating with trial-by-trial measures of CNV amplitude.

\section{Material and methods}

\section{Subjects}

Twelve healthy right-handed subjects (five females, seven males mean age 30 years) were recruited by advertisement and personal contact. All the subjects gave fully informed consent before the study. Ethical approval was obtained from the National Hospital for Neurology and Neurosurgery and the Institute of Neurology Joint Medical Ethics Committee.

\section{CNV task}

Subjects performed a forewarned reaction time task to elicit Contingent Negative Variation (CNV) during fMRI scanning. Subjects viewed a fixation cross at the center of a projection screen, and attended to auditory stimuli, presented via headphones in the scanner. The first warning tone, $\mathrm{S} 1=1000 \mathrm{~Hz}$, lasted $50 \mathrm{~ms}$ and was followed by the second imperative stimulus, S2 $=1000 \mathrm{~Hz}$, after a variable delay [mean $4 \pm 0.6$ $\mathrm{s}$ from the start of the beginning of the warning tone (S1)]. The loudness of S1 and S2 was set at $70 \mathrm{~dB}$. Subjects were required to make a reaction time button press response immediately after $\mathrm{S} 2$. The purpose of the variable delay in the $\mathrm{S} 1-\mathrm{S} 2$ interval was to induce a decorrelation of the timing of $\mathrm{CNV}$ potential and activity related to $\mathrm{S} 2$ and reaction-time response, while critically retaining the predictability of the interval (Jahanshahi et al., 1995). The task was optimized outside the scanner in two subjects to establish that psychological expectancy was retained and the morphology of the early component of $\mathrm{CNV}$ remained intact. Because of the temporal characteristics of the haemodynamic response function (HRF) in fMRI, decorrelation of CNVrelated BOLD activity from $\mathrm{S} 2$ and response-related activity is

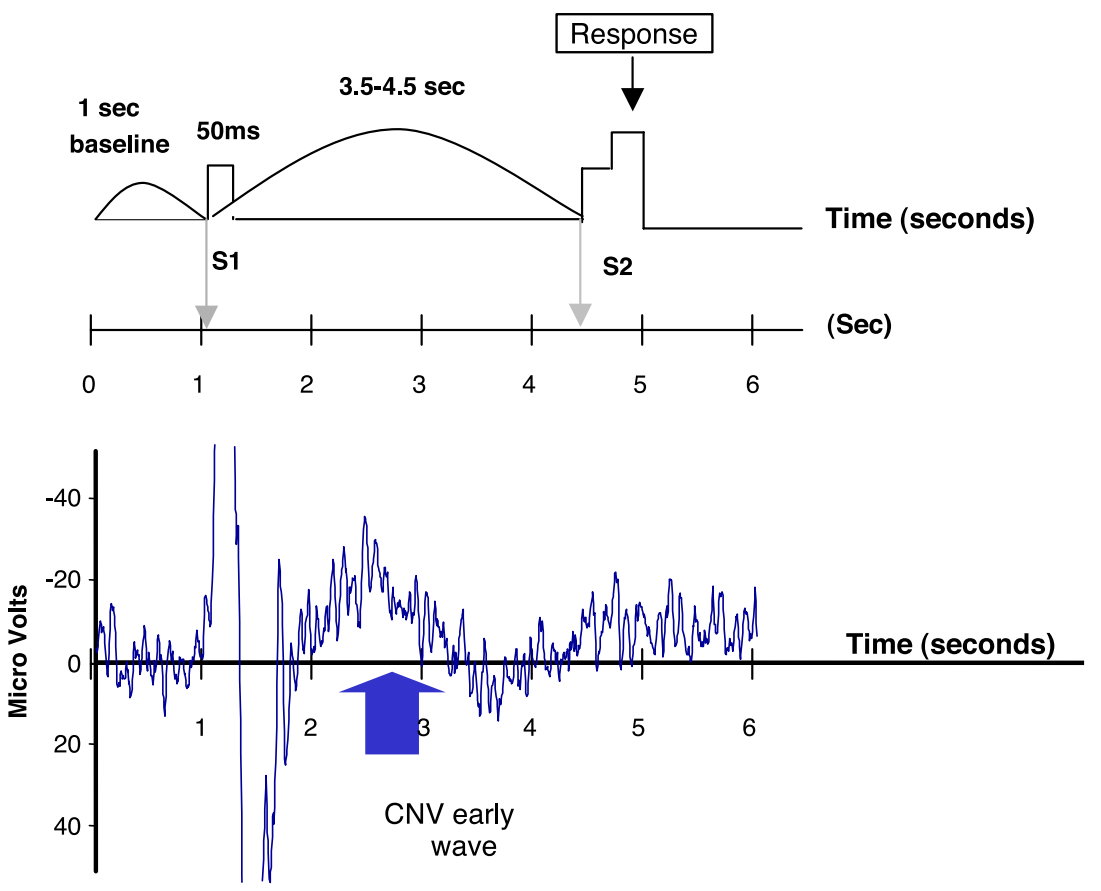

Fig. 1. Contingent negative variation paradigm and EEG data. Subjects performed two sessions of a forewarned reaction time task during fMRI data acquisition. Each subject, while visually fixating, was presented with repeated trials of two tones, an orienting (warning) tone (S1), and an imperative tone (S2), and was required to make a reaction-time (button-press) response to the S2. S2 occurred after a 3.5-4.5 s interval after the warning tone (S1). Contingent negative variation $(\mathrm{CNV})$ describes the slow negative shift in midline (vertex) scalp potential during the delay, initiated after S1 when the subject recognizes that the second stimulus (S2) is about to occur. This is illustrated diagrammatically in the upper figure. The variable (jittered) S1-S2 interval was used to deconfound motor responses from fMRI activity related to CNV. The averaged EEG data of one representative subject, obtained during simultaneous fMRI data acquisition are shown beneath. The orienting response to $\mathrm{S} 1$ is greatly enhanced in the scanner due to strong magnetic field. Constraints imposed by the time constant for EEG data acquisition resulted in relative suppression of CNV amplitude, yet the early phase of CNV is present, indicated by the blue arrow. Because the CNV data was averaged on the $\mathrm{S} 1$ stimulus, the later $\mathrm{CNV}$ component is effectively averaged out with responses relating to S2 and motor execution. 
acknowledged as only partial. This was maximized by modeling $\mathrm{CNV}$ as a fixed $3.5 \mathrm{~s}$ boxcar interval from $\mathrm{S} 1$, convolved with a fixed canonical HRF. The intertrial interval was randomly generated and lasted between 7 and $12 \mathrm{~s}$ (mean $8 \pm 1 \mathrm{~s}$ ).
During scanning, subjects underwent two sessions, each consisting of 40 trials (Fig. 1). Subjects were scanned twice with simultaneous EEG acquisition attempted during the second session.

A.
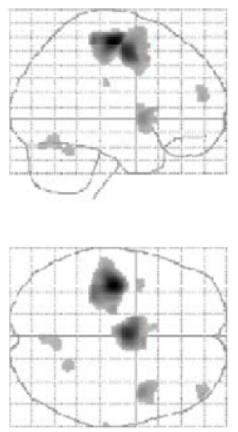

Glass

brain

projection

$<-2,-6,50$
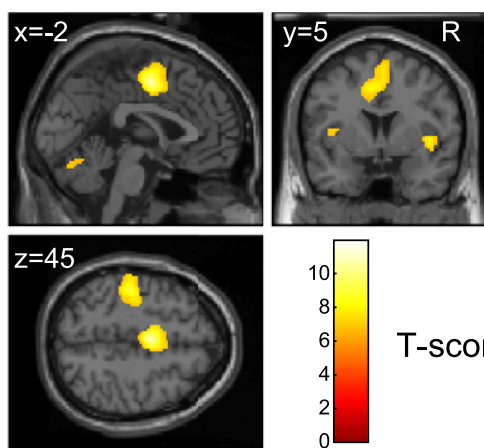

T-score

B.

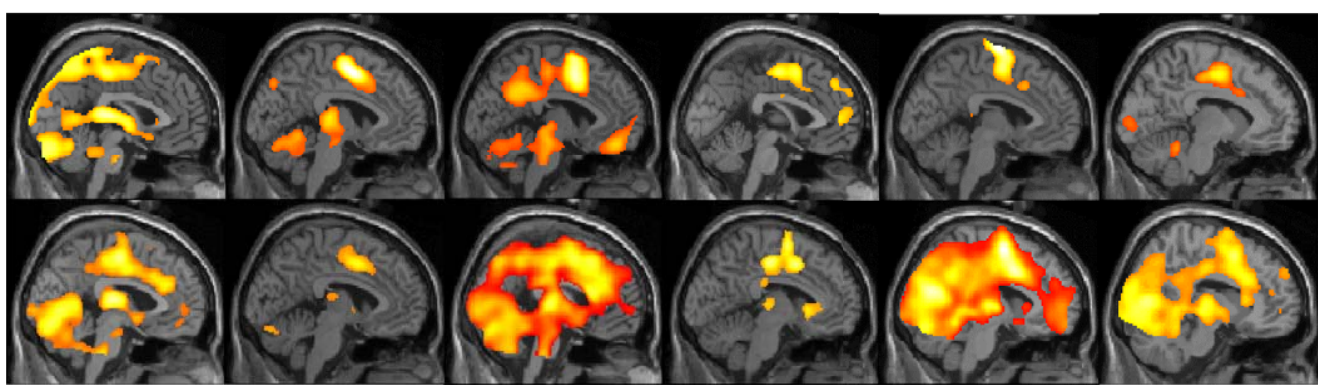

C.
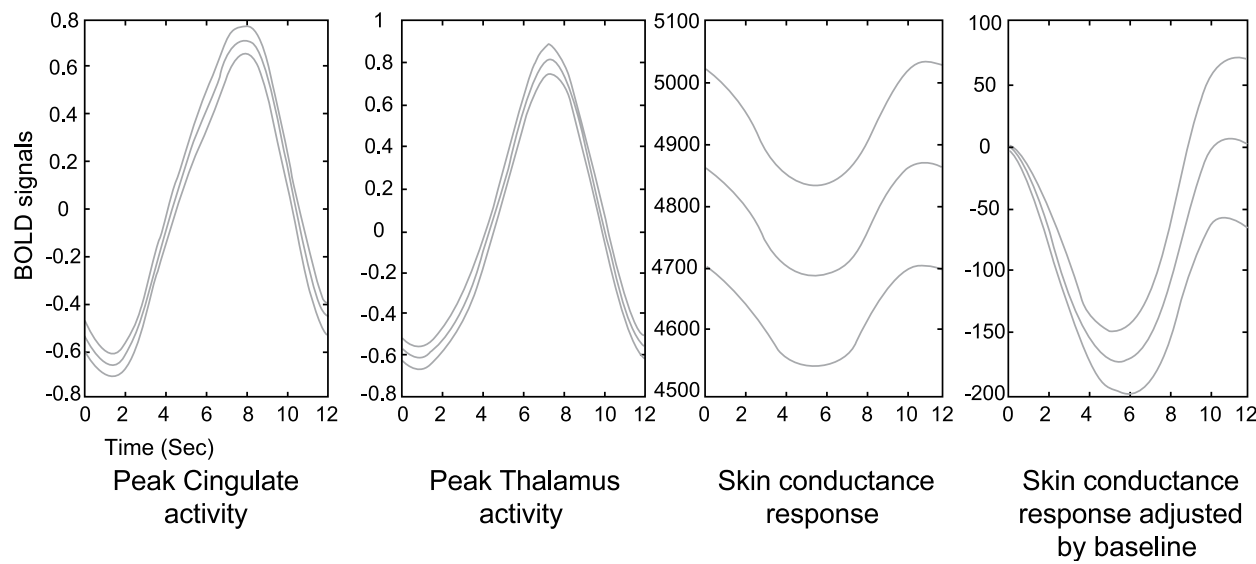

Fig. 2. (A) Group data for brain activity related to CNV. The figure illustrates group data associated with the main effect of CNV, that is, the 3.5-s epoch period following S1 presentation. To the left [N=12, random effects analysis (RFX), $P<0.05$, corrected], group activity is plotted on a glass brain projection, with a marker placed at the site of peak SMA/midcingulate activity ( $x, y, z$ coordinates given). Adjacent, the same data are plotted orthogonal sections of a normalized template brain to highlight location of activated regions, with a color bar indicating $t$ value of the effect and $x, y$, and $z$ coordinates given for each section. $\mathrm{R}=$ right hemisphere. CNV-related brain activity was observed midcingulate and adjacent supplementary motor cortex, left somatomotor cortex, bilateral insula, and dorsal midline cerebellum. (B) Individual data for brain activity related to CNV The figure illustrates individual data for the main effect of CNV-related brain activity plotted on sagittal sections of a template brain at $P<0.001$, uncorrected. In addition to the regions highlighted in A, the majority of subjects showed enhanced activity in dorsal thalamus, dorsal pons, basal ganglia, and perigenual cingulate. (C) Peak cingulate and thalamus activity during CNV task performance with accompanied GSR activity. The graphs illustrate data from one representative subject of the mean ( \pm standard error) time course of blood oxygenation level dependent (BOLD) activity during the CNV epoch in peak voxels located in the cingulate and thalamus, time locked to S1 presentation. Adjacent is plotted the mean ( \pm standard error) recorded electrodermal activity, averaged over trials and time locked in the same manner increased sympathetic arousal is shown in a positive direction. The graph on the right illustrates the averaged data corrected for GSR drift between trials. 


\section{Electrodermal monitoring}

Skin conductance (galvanic skin responses, GSR) was continuously measured (SCL 200, Biofeedback Systems, Manchester, UK) during the scanning using silver electrodes placed on subject's left index and middle fingers. Low-pass filtered skin conductance output was recorded using Spike 3 (CED, Cambridge, UK) with scanner synchronization pulses.

\section{Simultaneous EEG recording reconstruction and analysis}

In five subjects, we successfully obtained electroencephalography (EEG) simultaneously with acquisition of fMRI data. EEG was recorded using 10 channels. Gold electrodes fitted with $10 \mathrm{k} \Omega$ carbon resistors were applied on the scalp (Fpz, Fz, Cz, Pz, Oz, O1, $\mathrm{O} 2$, A1, and A2) according to the International 10/20 system for EEG. Two additional electrodes applied obliquely across the left eye detected eye blinks. The electrodes were connected to a headbox developed in-house (Allen et al., 1998, 2000), and placed close to the scanner bore. EEG signals were processed via a CED 1401 (Cambridge Electronic Design Limited, Cambridge, UK). EEG was recorded with 0.45 -s time constant and a sampling rate of $5000 \mathrm{~Hz}$, reduced to $200 \mathrm{~Hz}$ after image artifact reduction. In conventional EEG, optimal sampling rate is a minimum of three times the bandwidth. However, we required a sampling rate of $5000 \mathrm{~Hz}$ in the scanner environment to fully characterize and subsequently remove imaging-related artifacts. Imaging and pulse artifacts were processed using an established in-house EEG analysis system (Allen et al., 1998, 2000). High-frequency artifacts due to RF pulses were filtered before the main gain stage (Allen et al., 2000). For the CNV analysis, data were transferred to the Neuroscan Edit 4.3 (Neuroscan, USA). The magnitude of the CNV was calculated as the integral of CNV amplitude over $3.5 \mathrm{~s}$ following the first stimulus (S1), measured with respect to a baseline recorded for $1 \mathrm{~s}$ before $\mathrm{S} 1$ onset. A trial was rejected if eye movement or other artifacts exceeded $500 \mu \mathrm{V}$, and for the regression analysis, the $\mathrm{CNV}$ mean response was substituted as the value for that artifact trial. The later component of the $\mathrm{CNV}$, together with the S2 induced potential, was effectively averaged out because we included a variable interval between $\mathrm{S} 1$ and $\mathrm{S} 2$, and measured the $\mathrm{CNV}$ with respect to $\mathrm{S} 1$. The average $\mathrm{CNV}$, after the $\mathrm{DC}$ level had been removed, for 40 trials (one subject) is shown for one typical subject in Fig. 1 to highlight the reduced amplitude of the CNV signal during fMRI (estimated as a $30 \%$ reduction in signal to noise as a result of the short time constant) and the absence of a late component consequent upon the variable $\mathrm{S} 1-\mathrm{S} 2$ interval and short time constant.

\section{fMRI data acquisition}

Subjects were scanned during the CNV task using 2-T Siemens Magnetom VISION scanner using T2* echoplanar imaging weighted for blood oxygenation level dependent (BOLD) contrast [32 slices, flip angle $90^{\circ}$, echo time (TE) $40 \mathrm{~ms}$, TR, $2.4 \mathrm{~s}, 540$ acquisitions/session], and fMRI data were reconstructed using trajectory-based reconstruction (Josephs et al., 2000). During data acquisition, subjects were placed in the scanner with their head fixed to avoid head movement. The first six scans were discarded to allow T1 equilibration. Following acquisition of functional scans, a T1-weighted MPRAGE structural sequence $(\mathrm{TR} / \mathrm{TE}=$ $11 / 4 \mathrm{~ms}, \mathrm{TI} / \mathrm{TD}=1 / 0.5 \mathrm{~s}$ flip angle $12^{\circ}, 176$ sagittal slices) was taken from all subjects for anatomical registration with the functional BOLD data.

\section{fMRI data analysis}

Imaging data was processed and analyzed using Statistical Parametric Mapping (SPM99; Wellcome Department of Imaging Neuroscience, London, UK; http://www.fil.ion.ucl.ac.uk/spm/ spm99.html) (Friston et al., 1995a,b) to correct for head movement and for analyses of individual and group effects within the framework of the General Linear Model (Friston et al., 1995b, 1999). Design matrices in each subject were constructed modeling components of the $\mathrm{CNV}$ task together with continuous regressors for skin conductance. A mixed epoch and eventrelated model was used, in which the warning tone $\mathrm{S} 1$ and interval between the two tones were modeled together as a 3.5-s epoch that captured predominantly the early component of the $\mathrm{CNV}$. The imperative stimulus $\mathrm{S} 2$ and reaction time response was modeled separately as a delta function (event). Epochs and events were convolved with a canonical haemodynamic response function (HRF) without a temporal derivative. Skin conductance, an index of autonomic arousal, was modeled as a continuous regressor, without a delay, throughout task performance. Trialrelated changes in skin conductance were computed independently for illustrative purposes. Individual analyses were conducted to derive peak cingulate and thalamic activity associated with delay period of the CNV task (Fig. 2). Contrast images generated at the individual fixed effects level were analyzed subsequently using a second-level random effects approach (Friston et al., 1999).

We first tested for a significant main effect of the delay period (CNV-related epoch) of the CNV task in individual subjects and in a second-level group analyses. Next, to examine an association between enhanced SMA/midcingulate activity and enhanced activity in other brain regions, we used a univariate regression analysis of the peak SMA/midcingulate cluster in a second random effects analysis. To determine the relationship between delay period activity during CNV task performance and EEGderived measures of $\mathrm{CNV}$ amplitude, we performed a fixed effects

Table 1

Regional brain activity associated with the CNV

\begin{tabular}{lllr}
\hline Region & Side & $\begin{array}{l}\text { Coordinations } \\
\text { of peak activity }\end{array}$ & $T$ score \\
\hline Cingulate cortex + SMA & $\mathrm{L}$ & $-2,-6,50$ & $11.09^{*}$ \\
Somatomotor cortex & $\mathrm{L}$ & $-36,-14,60$ & $10.71^{*}$ \\
Insula & $\mathrm{R}$ & $42,6,0$ & $8.64^{*}$ \\
$\begin{array}{l}\text { Dorsolateral prefrontal } \\
\quad \text { cortex }\end{array}$ & $\mathrm{R}$ & $42,54,18$ & $7.62^{*}$ \\
Cerebellar vermis & - & $2,-74,-20$ & $7.58^{*}$ \\
Head of caudate & $\mathrm{L}$ & $-8,14,-2$ & $7.25^{*}$ \\
Insula & $\mathrm{L}$ & $-40,2,8$ & $7.23^{*}$ \\
Inferior parietal lobule & $\mathrm{R}$ & $66,-24,26$ & $7.12^{*}$ \\
Orbitofrontal cortex & $\mathrm{R}$ & $22,48,-12$ & $6.55^{*}$ \\
Occipitoparietal junction & $\mathrm{R}$ & $42,40,12$ & $6.47^{*}$ \\
Thalamus (lateral) & $\mathrm{L}$ & $-20,-18,10$ & $6.09^{\dagger}$ \\
Thalamus (pulvinar) & $\mathrm{R}$ & $12,-32,6$ & $5.43^{\dagger}$ \\
Thalamus (pulvinar) & $\mathrm{L}$ & $-10,-28,8$ & $5.38^{\dagger}$ \\
\hline
\end{tabular}

$* P<0.05$, corrected for whole brain.

${ }^{\dagger} P<0.05$, corrected for small volume of region of interest. 

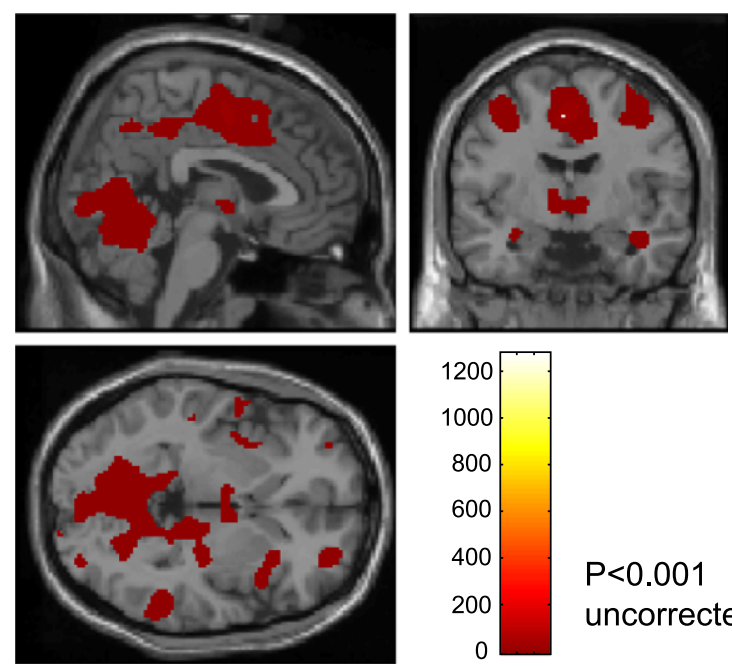

$\mathrm{P}<0.001$ uncorrected

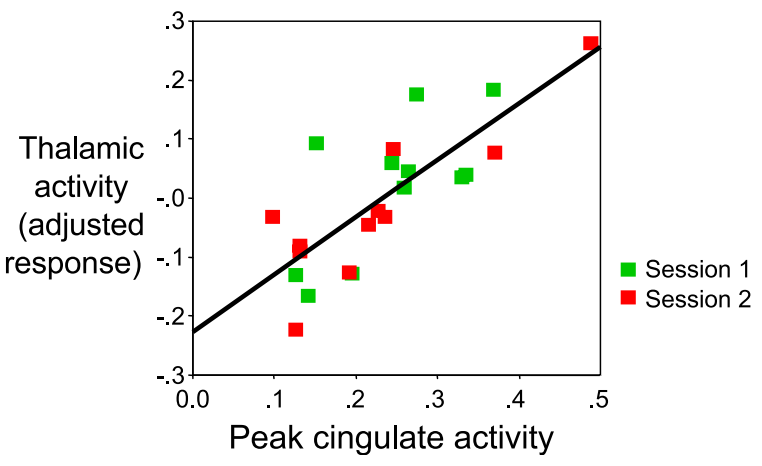

Fig. 3. Regional brain activity related to peak cingulate activity. Group data are plotted on normalized template brain, illustrating regional brain activity correlating across subjects with peak cingulate activity (at $P<0.001$, uncorrected). Brain regions most active when CNV-related cingulate activity was strongest include adjacent mid/anterior cingulate, the SMA, somatosensory hand area, hippocampus thalamus, and cerebellum. The color bar indicates the regional $t$ score. Data for the relationship between thalamic and cingulate activity strength, across subjects and sessions, is plotted in the graph below, illustrating this second-level correlation between thalamic and cingulate responses.

analysis of five subjects (five sessions) in whom EEG was successfully recorded during functional imaging. This analysis modeled parametric modulation of the epoch period by, trial-bytrial, measures of $\mathrm{CNV}$ amplitude. $F$-contrasts were used to determine areas showing a significant modulation by both main effects of delay period and amplitude of the CNV. Lastly, we assessed regional neural activity attributable to both changes in skin conductance and delay period activity, using conjunction analyses at the second level (with sphericity correction for repeated measures). Emphasis is given to data meeting threshold criteria of $P<0.05$, corrected for multiple comparisons across the whole brain using Family Wise Error, akin to a Bonferroni correction. For a priori regions of interest implicated in CNVrelated mechanisms, notably the thalamus, we performed a small volume correction for volume of interest (e.g., for thalamus a midline sphere: radius $20 \mathrm{~mm}$, center coordinates in $\mathrm{mm}$ from anterior commissure $0,-15,0$ ) (Worsley et al., 1996). Data are presented in figures at threshold of $P<0.001$, uncorrected for illustrative purposes.

\section{Results}

Neurophysiological effects of the forewarned reaction time task

The task successfully generated a CNV inside the scanner $(N=$ 5) of similar form to that piloted outside the scanner (Fig. 1). In spite of a short time constant for the CNV and an electrically noisy scanner environment, increased $\mathrm{CNV}$ amplitude was observed during interstimulus interval, compared to the baseline interval. The constraints of CNV acquisition within the fMRI environment was reflected in enhancement of the transient orienting response

A.
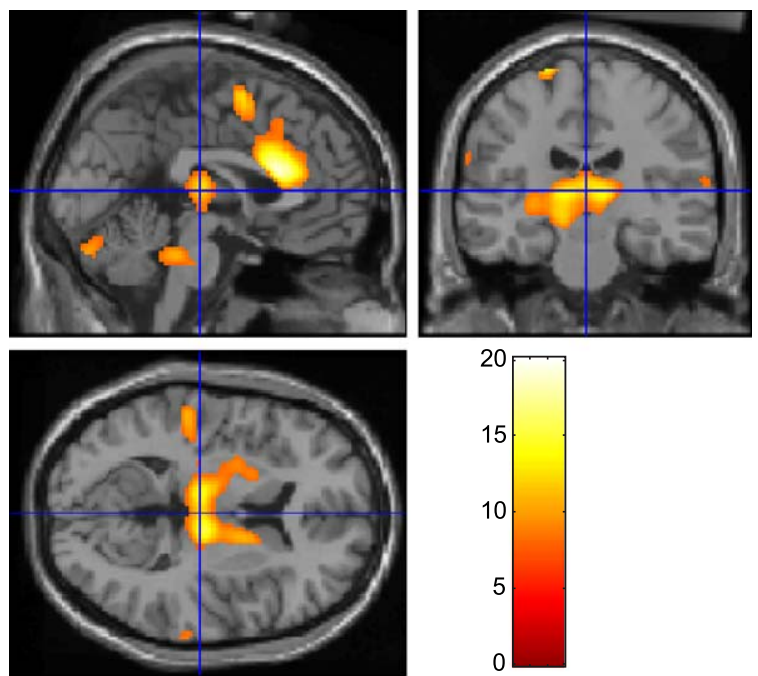

B.

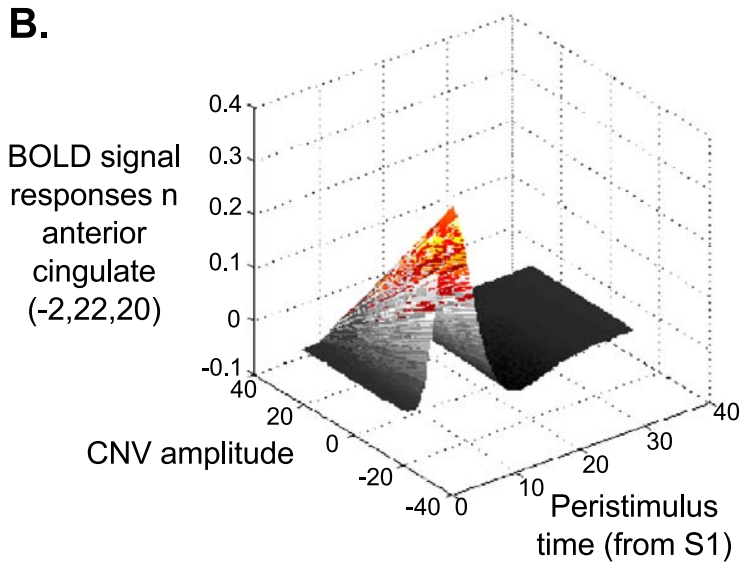

Fig. 4. (A) Brain regions modulated by $\mathrm{CNV}$ amplitude. In five subjects, $\mathrm{CNV}$ was recorded simultaneously with acquisition of fMRI data. A fixedeffect analysis was used to determine across these subjects regional brain activity correlating with trial-by-trial changes in measured CNV amplitude (derived as the integral over $3.5 \mathrm{~s}$ of baseline-corrected EEG data). $F$ tests of regions were activity-related significantly $(P<0.05$, corrected $)$ highlighted bilateral thalamus, anterior cingulate, SMA pons, and cerebellum. The distribution of this activity is plotted on orthogonal sections of a template brain, illustrating the location of thalamic involvement extending into basal ganglia. (B) Cingulate activity covarying with CNV amplitude. This figure illustrates positive covariation of anterior cingulate activity with the regressor for $\mathrm{CNV}$ amplitude. The modeled haemodynamic change is plotted as a function of time and the regressor for $\mathrm{CNV}$ amplitude for one subject. 
evoked by S1, which was further increased by pulse artifact. In the intense electromagnetic environment of the MR scanner, blood flow and pulsatile motion significantly effect EEG signals (Lemieux et al., 2001). No significant difference in CNV amplitude was observed between frontal $\mathrm{Fz}$ and central $\mathrm{Cz}$ electrodes, suggesting an equal contribution from sources of early and late components of the CNV. In most subjects, skin conductance activity decreased during anticipation of the $\mathrm{S} 2$ and showed subsequent rebound increase after the motor response to the imperative stimulus (Fig. 2C).

\section{Activity relating to the main effect of delay period}

To identify the functional neuroanatomy underlying generation of $\mathrm{CNV}$, we first tested for a significant main effect of delay period activity in the forewarned reaction time task $(3.5 \mathrm{~s}$ commencing at S1). Contrast images for the main effect of this epoch were obtained from each subject over two sessions and entered into second-level random effects analysis. At a stringent threshold ( $P<0.05$, corrected), enhanced activity related to the delay $\mathrm{CNV}$ period was observed in midcingulate cortex, adjacent supplementary motor cortex (SMA), left somatomotor cortex, (corresponding to the right hand area), bilateral insula, and dorsal midline cerebellum (Fig. 2A, Table 1). Individual subjects consistently demonstrated the above pattern of activity though there was individual variation in intensity of this to delay period activity. Enhanced activity in dorsal thalamus was also observed in group analysis at a threshold of $P<0.05$ (SVC) and in individual analyses was observed in the majority (8/12) of subjects (threshold of $P<0.001$, uncorrected), frequently associated with activity increases in dorsal pons, basal ganglia, and perigenual cingulate (Fig. 2B). In addition, significant $\mathrm{CNV}$ -
Skin conductance decreases
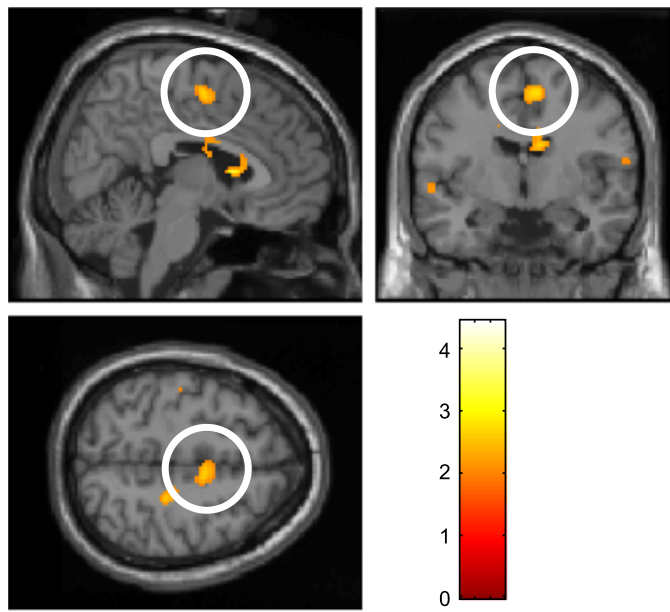

Skin conductance increases
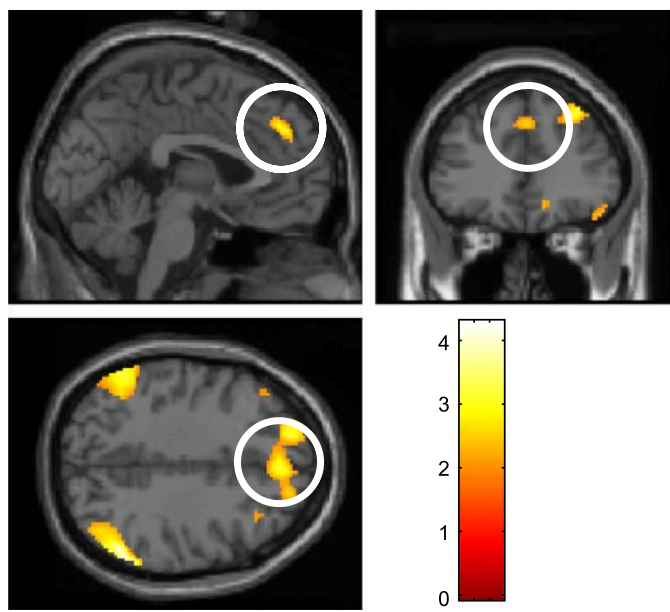
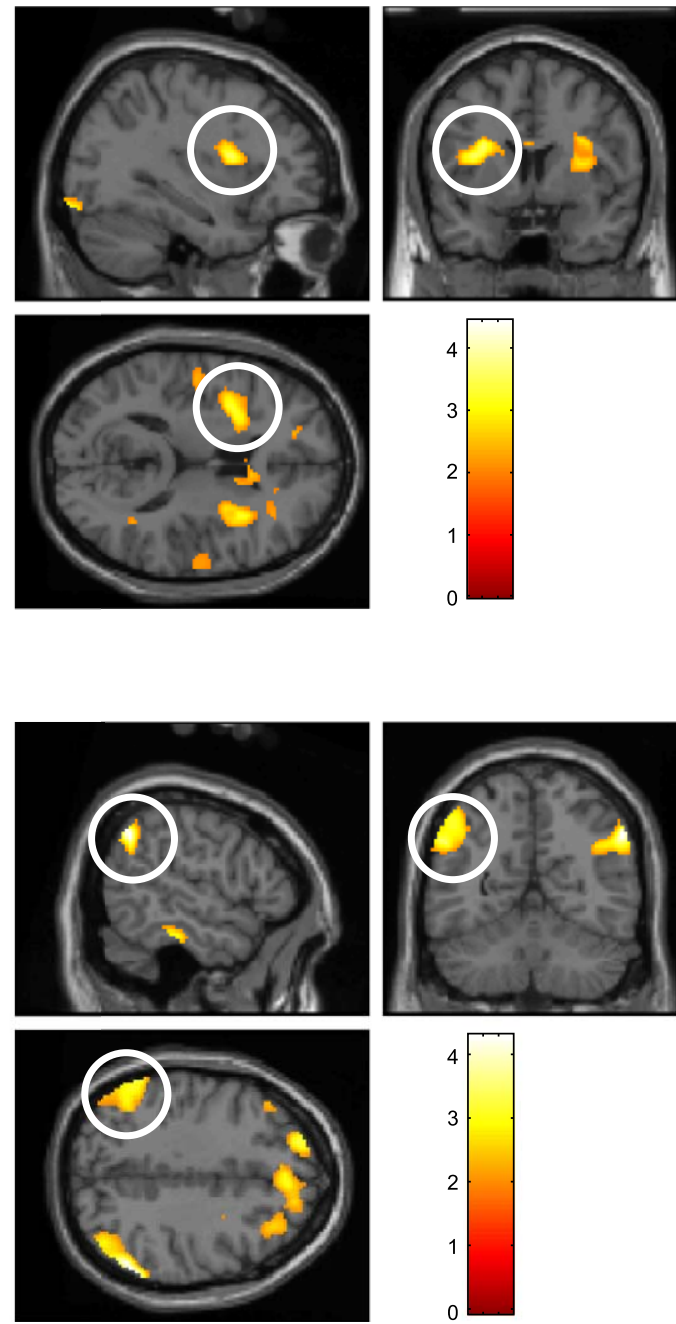

Fig. 5. CNV-related brain regions covarying with increases and decrease in sympathetic electrodermal arousal, indexed by GSR activity. Contrast images generated at the first level for activity relating to electrodermal activity and the main effect of CNV task epoch were entered into a second-level analysis. Using conjunction analysis to test for common effects, regions were identified where activity was significantly modulated by both the main effect of CNV and by changes in electrodermal arousal at a threshold of $P<0.001$, uncorrected. Regions sensitive to decreases in sympathetic activity and to the main effect of CNV included motor cingulate and bilateral anterior insula/operculum. Regional activity reflecting sensitivity to increasing sympathetic arousal and CNV included paracingulate/frontal polar cortices and bilateral parietal lobe. These activity patterns are illustrated on orthogonal sections of a template brain. 
related activity was observed in areas including dorsolateral prefrontal and parietal cortices, regions implicated in selective attentional processes. However, at an individual subject level, activity in prefrontal, orbitofrontal, and occipitoparietal cortices was less consistently observed.

\section{Correlation between cingulate and thalamus}

To explore the basis of individual differences in delay period activity observed in areas such as thalamus and basal ganglia, the magnitude of activity at the peak cingulate/SMA activity cluster was computed for each subject and used as a regressor of interest in a second-level regression analysis. This analysis allowed us to index, across subjects, functional associations between cingulate/SMA and other brain regions. We observed significant correlations between activity in dorsal cingulate and bilateral thalamus ( $P<0.05$, corrected) (Fig. 3). Additional areas that correlated with peak cingulate activity included adjacent regions of mid/anterior cingulate, supplementary motor area, somatosensory hand area, hippocampus, and cerebellum $(P<0.05$, corrected).

\section{Activity modulated by the CNV amplitude}

To identify brain activity directly related to CNV amplitude, we tested for a parametric modulation of activity during the interstimulus interval by the $\mathrm{CNV}$ amplitude using a fixedeffects analysis of data from the five subjects in whom $\mathrm{CNV} /$ EEG data was successfully recorded synchronously with fMRI. Each subject contributed one session of 40 trials with $\mathrm{CNV}$ amplitude integral employed as a regressor of interest. Delay period activity correlated with $\mathrm{CNV}$ amplitude was tested using an $F$ test with a threshold significance of $P<0.05$ (whole brain corrected). Activity within anterior cingulate $(-2,22,20)$, bilateral thalamus $(10,-18,8 ;-12,-20,8)$ and $\operatorname{SMA}(0,2$, 60) covaried with $\mathrm{CNV}$ amplitude $(P<0.05$ corrected) (Fig. 4A). The correlation between negative amplitude of the CNV and enhanced BOLD activity within the peak voxel identified in this analysis within anterior cingulate cortex is shown in Fig. 4B for one subject.

\section{Shared neuroanatomical substrate for $C N V$ and peripheral autonomic arousal}

We performed a second-level analysis of data from all 12 subjects to test the prediction that brain regions supporting $\mathrm{CNV}$ are modulated by peripheral autonomic arousal, indexed by GSR. Contrast images for activity attributable to GSR-related changes and the CNV-task delay period were entered into a second-level analysis to identify commonalities (i.e., where responses are modulated significantly by both GSR and the delay period associated with $\mathrm{CNV}$ ). One shortcoming is that this approach resulted in low-effect magnitude; hence, we report data at a reduced threshold ( $P<0.001$, uncorrected). CNV-related (delay period) activity associated with decreasing sympathetic arousal (it was found in the SMA/midcingulate region and bilateral insula). Within CNV-related brain regions, an area associated with increase in skin conductivity (increasing sympathetic arousal) was evident in paracingulate cortex, extending to frontal pole and in bilateral lateral parietal regions (Fig. 5).

\section{Discussion}

We investigated neural activity associated with CNV generation by measuring brain activity during performance of a forewarned reaction time task, a robust paradigm for eliciting CNV. By simultaneously recording EEG, in a subgroup of subjects, we were able to index regional brain activity associated with the trial-by-trial fluctuation in CNV amplitude, thereby identifying (and dissociating) activity related to CNV and from other task-related effects. A second methodological achievement entailed the simultaneous recording of GSR to identify sites of interface between two psychophysiological indices of attention (CNV) and arousal (GSR) (Frith and Allen, 1983).

Activity in supplementary motor area (SMA) and adjacent cingulate cortex, thalamus, and bilateral insula was enhanced during CNV task performance. We also noted effects in several other regions including parietal cortex, cerebellum, dorsolateral, and orbital prefrontal cortex. Some of this activity may reflect recruitment of regions involved in timing and in motor preparation during the delay period (Lee et al., 1999). However, the pattern of delay period activity was overshadowed by activation in the dorsal midline, close to the putative electrophysiological source of the CNV. Previous imaging studies using fMRI and MEG have implicated cingulate and SMA in both CNV and motor preparation (Ioannides et al., 1994; Lee et al., 1999; Liu et al., 1996). The SMA also contributes to coordination and programming of motor action, particularly when initiated voluntarily (Jankelowitz and Colebatch, 2002; Jenkins et al., 2000). Neuroanatomically, a functional circuit has been identified wherein the SMA sends efferent connections to the neostriatum, which in turn sends information back to the SMA via globus palidus and thalamus (Cunnington et al., 2001). Evidence for a role of SMA in motor preparation suggests that this region will have a greater contribution to late, rather than early, components of the CNV. Consistent with this proposal, a reduction in amplitude of CNV late component is reported in patients with Parkinson's disease in whom the integrity of the SMA-striatalthalamic circuit is disrupted (Ikeda et al., 1997). However, our own evidence, which preferentially highlights early components of the $\mathrm{CNV}$, suggests that SMA activity as well as that within cingulate cortex, may be reflected in both early and later phases of the CNV.

The early component of the CNV waveform, which has a peak at around $1 \mathrm{~s}$ after onset of the warning stimulus, reflects in part an orienting response and is influenced physical intensity of the S1 stimulus (Loveless and Sanford, 1975). An orienting response is defined as an automatic, preattentive change in central activity and peripheral state to novel and sudden environmental changes, closely linked to changes in autonomic activity (Sokolov, 1963). The design of our study was biased toward identifying activity related to this early $\mathrm{CNV}$ component. This was particularly apparent in the analysis of trial-by-trial covariation of regional brain activity with $\mathrm{CNV}$ amplitude where thalamic, cingulate, and insula activity was related to the early $\mathrm{CNV}$ component (the late component was not measured). It is noteworthy that these regions, in particular the anterior cingulate and insula, are implicated in representing and generating states of autonomic arousal that provide peripheral measures of stimulus orientation (Critchley et al., 2001, 2002, 2003).

Interestingly, the relationship between cingulate/SMA region and thalamic activity was highlighted by our findings of coincident delay period activity in both regions that covaried with the amplitude of the early phase of the CNV. The observation that 
magnitude of cingulate and thalamic activity during the delay period correlated across subjects provides additional evidence for a functional relationship between these regions. Enhanced activity within anterior cingulate cortex (ACC) is consistently observed in tasks that engage working memory, selective attention, response conflict monitoring, and during generation of error related negativity during executive functions (Barch et al., 2001; Braver et al., 2001; Luks et al., 2002). One influential proposal is that ACC activity plays a role as a detector of generic conflict (Braver et al., 2001; Carter et al., 2000) or signals the presence of high competition load between incompatible responses or information processes (Luks et al., 2002). Nevertheless, activity in ACC is also observed in low-level processes including regulation of peripheral arousal state and its integration with cognition (Critchley et al., $2000,2001,2003)$. In our study, we show that dorsal anterior cingulate activity relates to the preparatory delay period of the CNV task, with rostral ACC regions more associated with early, orienting components of the CNV. We also observed that ACC regions were influenced by peripheral autonomic arousal state of the subjects. These findings suggest that ACC activity observed during complex cognitive tasks may, at least in part, reflect lowlevel processes related to orientation, attentional, or autonomic engagement.

A major finding of our study is observed involvement of thalamus together with cingulate and SMA in CNV-related activity. Thalamic participation in CNV generation is suggested by neurophysiological observations describing the role of thalamic Reticular Nucleus in anticipatory attention (Birbaumer et al., 1990; Brunia and Van Boxtel, 2001; Elbert and Rockstroh, 1987; Skinner and Yingling, 1976). The thalamic reticular nucleus (RN) is proposed to regulate sensory information flow between thalamus and cortex via GABAergic interconnections supporting negative feedback in thalmo-cortical circuitry. In an attentive state, such as in the $\mathrm{CNV}$ task, activation of midbrain reticular centers decrease the firing of inhibitory thalamic RN interneurons, thereby enhancing information flow between thalamus and cortex and increasing CNV amplitude (Elbert and Rockstroh, 1987; Yingling and Skinner, 1975). In the awake state, enhanced attention increases both the linear transfer of sensory information from thalamus to cortex (Guillery et al., 1998) and the CNV-negative amplitude (Tecce, 1972). In addition to the role of RN neurons as a filter mechanism for sensory information (Yingling and Skinner, 1975, 1976), local inhibitory thalamic neurons in the mediodorsal nucleus and elsewhere in the thalamus, are critical to attention-related information processing in waking and attentive, vigilant state (Steriade, 2001, 2003). Our neuroimaging data implicate several thalamic regions, including mediodorsal nucleus in CNV-related activity. We would speculatively suggest that anterior cingulate cortex, where we observed BOLD activity correlated with negative amplitude of $\mathrm{CNV}$, represents the critical generator of the early phase of the $\mathrm{CNV}$. The greater correlation of (early phase) CNV amplitude with ACC, compared to SMA, activity, is consistent with the proposal that the potential is initiated in ACC activity (Liu et al., 1996; Luks et al., 2002). The thalamus, perhaps with basal ganglia, may act as a secondary regulator of anticipatory cortical activity and consequently contribute indirectly to the CNV negative potential. This assertion is consistent with the more anterior distribution of early phase, relative to the later phase, of CNV (Rohrbaugh et al., 1976). Moreover, the thalamus and other encapsulated subcortical structures are unlikely, anatomically, to directly contribute to generation of the CNV potential but may profoundly influence cortical excitation. The CNV may nevertheless represent a specific process common to preparatory attentional, sensory, and motor states. A close cognitive and neuroanatomical relationship has been reported between the $\mathrm{CNV}$ and the Bereitschaftspotential marking motor readiness (Jahanshahi et al., 1995). Nevertheless, patients with Parkinson's disease show reduced CNV amplitude but relatively intact Bereitschaftspotential, which lacks attentional component of the motor movement (Ikeda et al., 1997).

On a trial-by-trial basis, GSR activity, indexing peripheral sympathetic arousal, decreased over the S1-S2 interval and presentation and showed a rebound increase after execution of the motor response of the imperative S2 tone (Fig. 2). This observation suggests an inverse relationship may exist between $\mathrm{CNV}$ and peripheral arousal, consistent with the observation that increased heart rate is associated with $\mathrm{CNV}$ reduction (Tecce, 1972) and that there is deceleration of heart rate slows over the late phase of forewarned reaction time tasks such as that used to evoke CNV (Frith and Allen, 1983). The event-related GSR responses habituated over trials making the precise relationship between GSR and CNV-related BOLD responses difficult to determine. Nevertheless, conjunction analyses allowed us to identify discrete brain regions activated in common to the CNV task and sympathetic arousal. Enhanced activity in parietal and medial prefrontal cortical regions was common to both CNV task delay period and increasing sympathetic arousal. These regions are known to show activity enhancement during sustained attention tasks, suggesting that arousal level enhances this aspect of forewarned reaction time task performance. Conversely, CNV-related activity in midcingulate/SMA and bilateral basal ganglia was decreased with increased sympathetic arousal, consistent with the proposal that these motor preparatory areas are intimately linked with the representation of peripheral autonomic tone, and mediate the inverse relationship between central focused attention and bodily arousal state.

A number of technical compromises had to be made to achieve the objectives of our study. Simultaneous EEG and fMRI was successful in only five subjects and we used a shorter time constant for obtaining CNV data than would be used in an EEG laboratory setting. The experimental design was optimized to decorrelate the majority of the $\mathrm{S} 1-\mathrm{S} 2$ delay period (when $\mathrm{CNV}$ is generated) from S2 and response-related activity, while preserving the psychological expectancy of S2 (Jahanshahi et al., 1995). These two factors may have biased our findings toward regional activity related to the early phase of $\mathrm{CNV}$ and limited our inferences concerning the later CNV component. Nevertheless, our findings represent a unique, technically advanced and detailed identification of the functional neuroanatomy underlying the $\mathrm{CNV}$.

In summary, we identified a functional matrix of brain regions engaged during the delay period of a forewarned reaction time task associated with generation of the CNV. In particular, our study highlights contributions from SMA, cingulate, thalamus, and bilateral insula in anticipatory attention and motor preparatory processes engendered by the task that are indexed electrophysiologically by generation of the CNV. A crucial strength of our study is the simultaneous recording of CNV during fMRI data acquisition that enabled direct exploration of these electrophysiological and neurovascular correlates of "cortical arousal". Together, our findings confirmed involvement of thalamus, cingulate, and SMA in generation of CNV and supported model of attention and arousal mechanisms through regulation of sensory information between reentrant thalamocortical neural pathways. 


\section{Acknowledgments}

This work was supported by a Programme Grant to R.J.D. from the Wellcome Trust. Y.N. is supported by Raymond Way Fund. HC is supported by a Wellcome Clinician Scientist Fellowship. The help and advice of Drs. Pierre Maquet, Philip Allen, Louis Lemieux, Leun Otten, Angela Quayle, and Stefan Kiebel are gratefully acknowledged.

\section{References}

Allen, P.J., Polizzi, G., Krakow, K., Fish, D.R., Lemieux, L., 1998. Identification of EEG events in the MR Scanner: the problem of pulse artifact and a method for its subtraction. NeuroImage 8, 229-239.

Allen, P.J., Josephs, O., Turner, R., 2000. A method for removing imaging artifact from continuous EEG recorded during functional MRI. NeuroImage 12, 230-239.

Barch, D.M., Braver, T.S., Akbudak, E., Conturo, T., Ollinger, J., Snyder, A., 2001. Anterior cingulate cortex and response conflict: effects of response modality and processing domain. Cereb. Cortex 11, 837-848.

Birbaumer, N., Elbert, T., Canavan, A.G.M., Rockstroh, B., 1990. Slow potentials of cerebral cortex and behavior. Physiol. Rev. 70, 1-41.

Braver, T.S., Barch, D.M., Gray, J.R., Molfese, D.L., Snyder, A., 2001. Anterior cingulate cortex and response conflict: effects of frequency, inhibition and errors. Cereb. Cortex 11, 825-836.

Brunia, C.H.M., Van Boxtel, G.J.M., 2001. Wait and see. Int. J. Psychophysiol. 43, 59-75.

Carter, C.S., Macdonald, A.M., Botvinick, M., Ross, L.L., Stenger, V.A., Noll, D., Cohen, J.D., 2000. Parsing executive processes: strategic vs. evaluative functions of the anterior cingulate cortex. PNAS 97, $1944-1948$.

Critchley, H.D., Melmed, R.N., Featherstone, E., Mathias, C.J., Dolan, R.J., 2000. Brain activity during biofeedback relaxation. A functional neuroimaging investigation. Brain 124, 1003-1012.

Critchley, H.D., Mathias, C.J., Dolan, R.J., 2001. Neural activity in the human brain relating to uncertainty and arousal during anticipation. Neuron 29, 537-545.

Critchley, H.D., Melmed, R.N., Featherstone, E., Mathias, C.J., Dolan, R.J., 2002. Volitional control of autonomic arousal: a functional magnetic resonance study. NeuroImage 16, 909-919.

Critchley, H.D., Josephs, O., O’Doherty, J., Zanini, S., Dewar, B.-K., Mathias, C.J., Cipolotti, L., Shallice, T., Dolan, R.J, 2003. Human cingulate cortex and autonomic cardiovascular control: Converging neuroimaging and clinical evidence. Brain 126, 2139-2152.

Cunnington, R., Egan, G.F., O'Sullivan, J.D., Hughes, A.J., Bradshaw, J.L., Colebatch, J.G., 2001. Motor imagery in Parkinson's disease: a PET study. Mov. Disord. 16, 849-857.

Elbert, T., Rockstroh, B., 1987. Threshold regulation-A key to the understanding of the combined dynamics of EEG and event-related potentials. J. Psychophysiol. 4, 317-333.

Frith, C.D., Allen, H.A., 1983. The skin conductance orienting response as an index of attention. Biol. Psychol. 17, 27-39.

Friston, K., Ashburner, J., Frith, C.D., Poline, J.B., Heather, J.D., Frackowiak, R.S., 1995a. Spatial registration and normalization of images. Hum. Brain Mapp. 2, 165-210.

Friston, K.J., Holmes, A., Worsley, K.J., Poline, J.P., Frith, C.D., Frackowiak, R.S., 1995b. Statistical parametric maps in functional imaging: a general linear approach. Hum. Brain Mapp. 2, 189-210.

Friston, K.J., Holmes, A.P., Price, C.J., Buchel, C., Worsley, K.J., 1999. Multisubject fMRI studies and conjunction analyses. NeuroImage 10, 385-396.

Guillery, R.W., Feig, S.L., Lozsadi, D.A., 1998. Paying attention to the thalamic reticular nucleus. Trends Neurosci. 21, 28-32.
Ikeda, A., Shibasaki, H., Kaji, R., Terada, K., Nagamine, T., Honda, M., Kimura, J., 1997. Dissociation between contingent negative variation $(\mathrm{CNV})$ and Bereitschaftspotential (BP) in patients with parkinsonism. Electroencephalogr. Clin. Neurophysiol. 102, 142-151.

Ioannides, A.A., Fenwick, P.B., Lumsden, J., Liu, M.J., Bamidis, P.D., Squires, K.C., Lawson, D., Fenton, G.W., 1994. Activation sequence of discrete brain areas during cognitive processes: results from magnetic field tomography. Electroencephalogr. Clin. Neurophysiol. 91, $399-402$.

Jahanshahi, M., Jenkins, I.H., Brown, R.G., Marsden, C.D., Passingham, R.R., Brooks, C.D., 1995. Self-initiated versus externally triggered movements I. An investigation using measurement of regional cerebral blood flow with PET and movement-related potentials in normal and Parkinson's disease subjects. Brain 118, 913-933.

Jankelowitz, S.K., Colebatch, J.G., 2002. Movement-related potentials associated with self-paced, cued and imagined arm movements. Exp. Brain. Res. 147, 98-107.

Jenkins, I.H., Jahanshahi, M., Jueptener, M., Passingham, R.E., Brooks, D.J., 2000. Self-initiated versus externally triggered movements: II. The effect of movement predictability on regional cerebral blood flow. Brain $123,1216-1228$.

Josephs, O., Deichmann, R., Turner, R., 2000. Trajectory measurement and generalized reconstruction in rectilinear EPI. Proc. Int. Soc. Magn. Reson. Med. 8, 1517.

Le Masson, G., Renaud-Le Masson, S., Debay, D., Bal, T., 2002. Feedback inhibition controls spike transfer in hybrid thalamic circuits. Nature $417,854-858$.

Lee, K.M., Chang, K.H., Roh, J.K., 1999. Subregions within the supplementary motor area activated at different stages of movement preparation and execution. NeuroImage 9, 117-123.

Lemieux, L., Salek-Haddadi, A., Josephs, O., Allen, P., Toms, N., Scott, C., Krakow, K., Turner, R., Fish, D.R., 2001. Event-related fMRI with simultaneous and continuous EEG: description of the method and initial case report. NeuroImage 14, 780-787.

Liu, M.J., Fenwick, P.B.C., Lumsden, J., Lever, C., Stephan, K., Ioannides, A.A., 1996. Averaged and single-trial analysis of cortical activation sequences in movement preparation, initiation and inhibition. Hum. Brain Mapp. 4, 254-264.

Loveless, N.E., Sanford, A.J., 1975. The impact of warning signal intensity on reaction time and components of the contingent negative variation. Biol. Psychol. 2, 217-226.

Luks, T.L., Simpson, G.V., Feiwell, R.J., Miller, W.L., 2002. Evidence for anterior cingulate cortex involvement in monitoring preparatory attentional set. NeuroImage 17, $792-802$.

Rockstroh, B., Elbert, T., Lutzenberger, W., Altenmuller, E., 1991. Effect of the anticonvulsant benzodiazepine clonazepam on event-related brain potentials in humans. Electroencephalogr. Clin. Neurophysiol. 78, $142-149$.

Rohrbaugh, J.W., Syndulko, K., Lindsley, D.B., 1976. Brain wave components of the contingent negative variation in humans. Science 191, $1055-1057$.

Skinner, J.E., Yingling, C.D., 1976. Regulation of slow potential shifts in nucleus reticularis thalami by the mesencephalic reticular formation and the frontal granular cortex. Electroencephalogr. Clin. Neurophysiol. 40, $288-296$.

Sokolov, E.N., 1963. Perception and the Conditioned Reflex. Pergamon, Oxford.

Steriade, M., 2001. Impact of network activities on neuronal properties in corticothalamic systems. J. Physiol. 86, 1-39.

Steriade, M., 2003. Presynaptic dendrites of thalamic local-circuit neurons and sculpting inhibition during activated states. J. Physiol. 546, 1.

Tecce, J.J., 1972. Contingent negative variation (CNV) and psychological processes in man. Psychol. Bull. 77, 73-108.

Walter, W.G., Cooper, R., Aldridge, V.J., McCallum, W.C., Winter, A.L., 1964. Contingent negative variation. An electric sign of sensorimotor association and expectancy in the human brain. Nature 203, $380-384$. 
Worsley, K.J., Marrett, S., Neelin, P., Vandal, A.C., Friston, K., Evans, A.C., 1996. A unified statistical approach for determining significant signals in images of cerebral activation. Hum. Brain Mapp. 4, 58-73.

Yingling, C.D., Skinner, J.E., 1975. Regulation of unit activity in nucleus reticularis thalami by the mecensephalic reticular formation and the frontal granular cortex. Electroencephalogr. Clin. Neurophysiol. 39, $635-642$.

Yingling, C.D., Skinner, J.E., 1976. Selective regulation of thalamic sensory relay nuclei by nucleus reticularis thalami. Electroencephalogr. Clin. Neurophysiol. 41, 476-482. 